Research Paper

\title{
Effect of alcohols on filamentation, growth, viability and biofilm development in Candida albicans
}

\author{
Nitin M Chauhan, Ravikumar B Shinde, S. Mohan Karuppayil \\ DST-FIST and UGC-SAP Sponsored School of Life Sciences, SRTM University, Nanded, M.S, India.
}

Submitted: August 2, 2012; Approved: April 4, 2013.

\begin{abstract}
In this study we report the potential of alcohols as morphogenetic regulators in Candida albicans. All the alcohols tested influenced various modes of growth like planktonic as well as biofilm forms. Viability was affected at high concentrations. Among the alcohols, the response of C. albicans to amyl alcohol (pentanol) was noteworthy. Amyl alcohol at a concentration $0.5 \%$ which was not inhibitory to growth and viability specifically inhibited morphogenetic switching from yeast to hyphal forms. It also inhibited normal biofilm development favoring yeast dominated biofilms. Based on this study we hypothesize that alcohols produced under anaerobic conditions may not favor biofilm development and support dissemination of yeast cells. Since anaerobic conditions are not found to favor production of quorum sensing molecules like farnesol, the alcohols may play a role in morphogenetic regulation.
\end{abstract}

Key words: alcohols, signalling, morphogenesis, biofilms, yeast metabolism, Candida albicans.

\section{Introduction}

Candida albicans is considered as a serious pathogen in immunocompromised patients causing high mortality rate (Fridkin and Irwin, 1983; Jean-Marcel, 1998; Virudes et al., 2003; Parahym et al., 2009). Several studies have shown that in C. albicans, filamentation is a virulence factor (Lo et al., 1997; Saville et al., 2003; Yang, 2003). It also plays a critical role in biofilm formation (Baillie and Douglas, 1999). Thus, inhibition of filamentation may reduce pathogenesis and biofilm formation in C. albicans (Lo et al., 1997; Baillie and Douglas, 1999; Lopez-Ribot, 2005).

Metabolites produced under different environmental conditions are reported as virulence molecules in microbes (Dufour and Rao, 2011). Different alcohols are produced by the yeast Saccharomyces cerevisiae and C. albicans in vitro (Ghosh et al., 2008; Hazelwood et al., 2008). For example in presence of leucine, valine, phenylalanine, tyrosine, and tryptophan as nitrogen sources, Saccharomyces cerevisiae produces isoamyl alcohol, isobutanol, tyrosol, phenylethanol, and tryptophol respectively (Dickinson, 2008; Hazelwood et al., 2008). Alcohols such as ethanol, propanol, isopropanol, 1-butanol, 2-butanol, isoamyl alcohol, and tert-amyl alcohol are reported to induce fila- mentation in S. cerevisiae (Lorenz et al., 2000). Attachment to tissue culture dishes and polystyrene dishes in Pseudomonas species is affected by methanol, ethanol, propanol, and butanol (Fletcher, 1983). C. albicans in presence ethyl alcohol, dodecanol, isoamyl alcohol, and nerolidol failed to switch from yeast to hyphae under standard induction conditions (Martins et al., 2007; Davis-Hanna et al., 2008; Chauhan et al., 2010). Short chain n-alkanols are reported to affect the morphogenesis in the dimorphic fungus, Aureobasidium pullulans (Moragues et al., 1998). Studies are lacking on the effect of propanol, butanol, isopropanol, pentanol and isobutanol in C. albicans. In this paper we report for the first time the effect of short chain alcohols on induced morphogenesis, growth, and biofilm formation in C. albicans.

\section{Materials and Methods}

\section{Organism, media and culture conditions}

Candida albicans ATCC 90028 (MTCC 3017) was used throughout the study. The culture was maintained on Yeast-Peptone-Dextrose (YPD) agar slants at $4{ }^{\circ} \mathrm{C}$. YeastPeptone-Dextrose medium (YPD) was prepared by dissolving individual components (Yeast extract 1\%, Peptone 2\% 
and Dextrose 2\%) in distilled water. $\mathrm{pH}$ was adjusted to 6.5. Solid medium was prepared by adding $2.5 \%$ agar powder to YPD broth. Two inducers i.e. $10 \%$ horse serum was prepared in deionized distilled water while, RPMI -1640 medium with L-glutamine w/o sodium bicarbonate buffered with $165 \mathrm{mM}$ MOPS (3-[N-morpholine] propane sulphonic acid) was added in sterilized distilled water and $\mathrm{pH}$ was adjusted to 7. All the media components and chemicals were from Hi-Media Laboratories Ltd. Mumbai, India. For culture activation, a single colony from the YPD plates was inoculated in $50 \mathrm{~mL}$ of YPD broth, in a $250 \mathrm{~mL}$ conical flask and incubated at $30^{\circ} \mathrm{C}$ on an orbital shaker at $120 \mathrm{rpm}$ for $24 \mathrm{~h}$. Cells from the activated culture were harvested by centrifugation for $5 \mathrm{~min}$ at $2000 \mathrm{~g}$ speed and washed three times with PBS (10 mM Phosphate buffer, $2.7 \mathrm{mM}$ Potassium chloride and $137 \mathrm{mM}$ Sodium chloride $\mathrm{pH}$ 7.4) and resuspended in PBS.

\section{Filamentation assay}

Filament formation was studied using microtiter plate assay in 96 -well microtiter plates as described previously (Chauhan et al., 2010). Briefly, cells from stock were inoculated in various inducer media to get $1 \times 10^{6}$ cells ml- . Various concentrations of butanol, isobutanol, tertiary-butanol, propanol, isopropanol and pentanol to give final concentrations of $0.06,0.12,0.25,0.5,1,2$, and $4 \%(\mathrm{v} / \mathrm{v})$ were added. Wells without alcohol were kept as control. Final volume of assay system in each well was kept $200 \mu \mathrm{L}$. The plates were incubated at $37{ }^{\circ} \mathrm{C}$ at $200 \mathrm{rpm}$ on an orbital shaker for 4 to $8 \mathrm{~h}$. After incubation, cells were observed microscopically. Every time, 100 cells were counted and the numbers of yeast and germ tube forms were noted. Percentage of germ tube formation in each well was calculated compared to that of control with the following formula:

$$
\% \text { formation }=\frac{\text { no. treatment }}{\text { no. } \text { control }} \times 100
$$

where $\%$ formation $=$ percentage of germ tube formation, no. treatment $=$ no. of germ tubes in treatment and no. control $=$ no. of germ tubes in control.

\section{Growth and viability assay}

Effect of alcohols on growth of C. albicans yeast phase cells was determined by standard CLSI M27-A microbroth dilution method (NCCLS, 1997). Various concentrations of butanol, isobutanol, tertiary-butanol, propanol, isopropanol, and pentanol (amyl alcohol) ranging from 0.025 to $4 \% \mathrm{v} / \mathrm{v}$ were added to RPMI 1640 medium in 96-well microtiter plates. Each well was inoculated with $1 \mathrm{x}$ $10^{3}$ cells $\mathrm{mL}^{-1}$. Final volume in each well was kept as $200 \mu \mathrm{L}$. Plates were incubated at $35^{\circ} \mathrm{C}$ on an orbital shaker for $24 \mathrm{~h}$. Optical density was measured at $620 \mathrm{~nm}$ wavelength using a 96-well microplate reader (Multiscan Ex-Thermo Electron Corp. USA) and absorbance of alcohols treated wells were compared with that of control. Plate count was done to see the effect on viability (Chauhan et al., 2010). Cells from the respective wells were diluted to get 300 to 400 colonies and an aliquot of the sample was spread on YPD agar plates. The plates were incubated at $30^{\circ} \mathrm{C}$ for $24 \mathrm{~h}$ and colony count was done. Percentage of viability of treated cells was calculated by comparing with the colony count of control wells.

\section{Biofilm formation and MTT assay}

To see the effect of various alcohols on biofilm development, Candida biofilms were developed on polystyrene surface of 96-well plates(Chauhan et al., 2010). A cell suspension of $1 \times 10^{7}$ cells $\mathrm{mL}^{-1}$ was prepared in PBS and $100 \mu \mathrm{L}$ was inoculated in each well. The plates were incubated at $37^{\circ} \mathrm{C}$ at $50 \mathrm{rpm}$ for $90 \mathrm{~min}$ for adhesion of cells on the surfaces. Non-adhered cells were removed by washing the wells, 2-3 times with PBS after 90 min. $200 \mu \mathrm{L}$ of RPMI-1640 medium along with various concentrations of butanol, isobutanol, tertiary-butanol, propanol, isopropanol, and pentanol ranging from 0.025 to $4 \% \mathrm{v} / \mathrm{v}$ and one control without alcohols was added to each well. The plates were incubated at $37^{\circ} \mathrm{C}$ for $24 \mathrm{~h}$ to allow biofilm formation. After incubation, wells were washed to remove any planktonic cells and biofilms were observed under an inverted light microscope (Metzer, India). Measurement of metabolic activity of biofilm was done by MTT assay with little modification (Hawser and Douglas, 1997). The tetrazolium salt 3-[4, 5-dimethylthiazol-2-yl]-2, 5-diphenyltetrazolium bromide (MTT, Hi-media, Mumbai, India) was used in an assay. $50 \mu \mathrm{L}$ of MTT solution (stock solution containing $5 \mathrm{mg} \mathrm{MTT}^{-\mathrm{ml}}$ of PBS, diluted 1:5 in prewarmed $0.15 \mathrm{M} \mathrm{PBS}$ prior to addition) was added in each well. The plate was incubated for $5 \mathrm{~h}$ at $37^{\circ} \mathrm{C}$. Dimethyl sulfoxide $(200 \mu \mathrm{L})$ was added to each well to solubilise MTT formazan product and optical density was measured at $450 \mathrm{~nm}$ using a 96-well microplate reader (Multiscan Ex-Thermo Electron Corp. USA). Percentage of metabolic activity in biofilm formation was calculated compared to that of control (without alcohol).

\section{Statistical analysis}

All the experiments were done in triplicates and standard deviation from the mean was calculated. Effect of alcohols on biofilm formation was analyzed using Two-way Anova (Bonferroni posttests) by GraphPad Prism 5 software. $\mathrm{P}<0.05$ was considered statistically significant.

\section{Results}

\section{Effect of various alcohols on induced morphogenesis}

All the alcohols inhibited filamentation in a concentration dependent manner induced by two standard inducers (Figure 1). Responses of C. albicans to alcohols were found to be similar for both the inducers at all time points studied. Pentanol at a concentration of $0.5 \%$ showed com- 
plete inhibition of filamentation, while $1 \%$ of butanol and propanol completely blocked hyphae. Isobutanol, isopropanol and tertiary-butanol at $2 \%$ completely halted yeast to hyphal form transitions (Figure 3). The efficacy of alcohols towards induced morphogenesis is as follows:

\section{Pentanol $<$ Butanol, Propanol $<$ Isobutanol, Isopropanol, Tertiary-Butanol}

\section{Growth and viability of $C$. albicans cells in presence of alcohols}

Alcohols upto $0.5 \%$ of concentration did not cause any effect on viability. Addition of $4 \%$ of various alcohols inhibited growth by $60-80 \%$. $2 \%$ of butanol, isobutanol, tertiary-butanol, and pentanol reduced growth by $15-20 \%$, whereas propanol and isopropanol at the same concentration inhibited growth by $20-25 \%$. $4 \%$ of butanol, isobutanol, and propanol were found to be toxic to Candida cells, while isopropanol, tertiary-butanol, and pentanol caused $35-50 \%$ reduction in viability at $4 \%$ (Table 1 ). The sensitivity of Candida cells for alcohols is as follows:

\section{Butanol $<$ Propanol $<$ Isobutanol $<$ Isopropanol $<$ Pentanol $<$ Tertiary-butanol}

\section{Candida biofilms in presence of various alcohols}

All the alcohols studied significantly $(p=0.0005)$ inhibited biofilm formation in a concentration dependent manner. Treatment with $4 \%$ of alcohols caused $50-60 \%$ reduction in metabolic activity in Candida biofilm (Figure 2). Addition of $2 \%$ of butanol, isobutanol, tertiary-butanol, propanol, and isopropanol after adhesion phase inhibited biofilm development and patches of few adhered yeast cells were observed, whereas similar effect was seen at $1 \%$ of pentanol (Figure 4). 1\% of butanol, isobutanol, tertiarybutanol, propanol, and isopropanol inhibited filamentation in biofilms and favoured 'yeast only' biofilm. Pentanol caused considerable inhibition of biofilm at $0.5 \%$ concentration leading to yeast only biofilm formation.
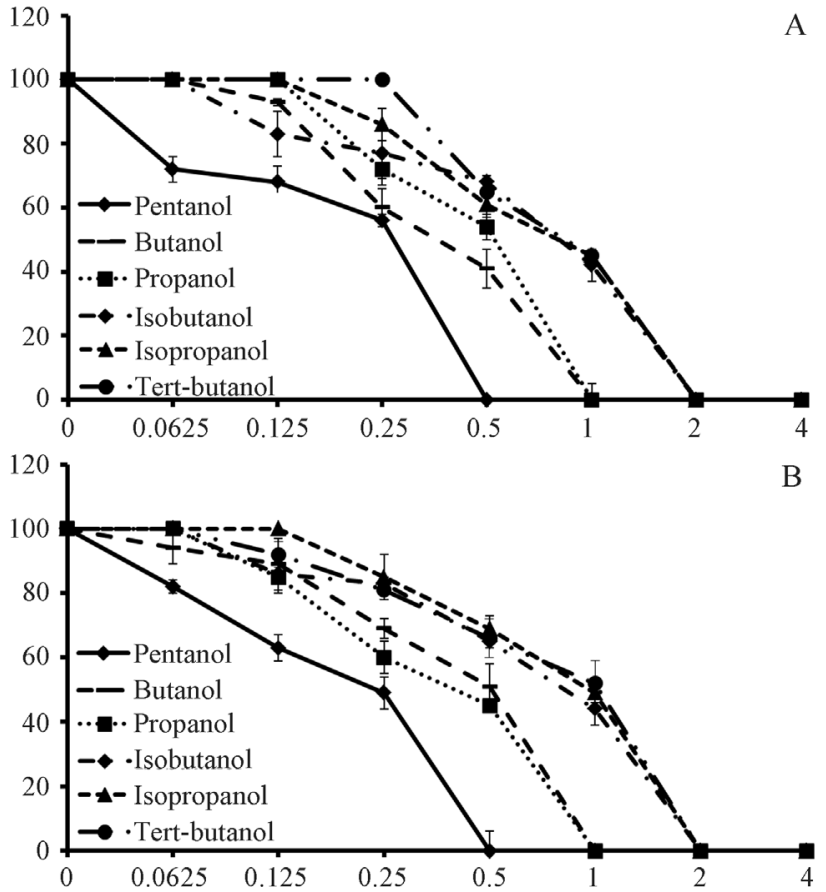

Figure 1 - Candida albicans yeast to hyphal form transition in presence of various alcohols induced by two standard inducers. A) Serum; B) RPMI-1640 medium. The cells were incubated at $37^{\circ} \mathrm{C}$ in respective medium for $4 \mathrm{~h}$ and morphology was assessed after incubation.

\section{Discussion}

The behavior and production of metabolites by Candida albicans in vivo may greatly vary depending on the niches it colonizes in the human body like gastrointestinal tract, vaginal, or oral cavities (Ghosh et al., 2008). In deep seated mycosis or in biofilms on prosthetic devices it may live in a polymicrobial environment and may be subject to anaerobic conditions or exposed to the metabolites its own or that of other organisms (Dumitru et al., 2004; Dufour and Rao, 2011). In gastrointestinal tract, deep

Table 1 - Viability of Candida albicans yeast phase cells grown in presence of alcohols

\begin{tabular}{|c|c|c|c|c|c|c|}
\hline \multirow{2}{*}{$\begin{array}{l}\text { Concentrations of } \\
\text { alcohols \% (v/v) }\end{array}$} & \multicolumn{6}{|c|}{$\%$ Viability } \\
\hline & Propanol & Isopropanol & Butanol & Isobutanol & Tert-butanol & Pentanol \\
\hline 0 & $100( \pm 0)$ & $100( \pm 0)$ & $100( \pm 6)$ & $100( \pm 0)$ & $100( \pm 0)$ & $100( \pm 0)$ \\
\hline 0.062 & $100( \pm 0)$ & $100( \pm 0)$ & $100( \pm 6)$ & $100( \pm 0)$ & $100( \pm 0)$ & $100( \pm 0)$ \\
\hline 0.125 & $100( \pm 0)$ & $100( \pm 1)$ & $100( \pm 3)$ & $100( \pm 0)$ & $100( \pm 1)$ & $100( \pm 1)$ \\
\hline 0.25 & $100( \pm 0)$ & $100( \pm 2)$ & $100( \pm 4)$ & $100( \pm 1)$ & $100( \pm 3)$ & $100( \pm 9)$ \\
\hline 0.5 & $100( \pm 1)$ & $100( \pm 3)$ & $100( \pm 5)$ & $100( \pm 9)$ & $100( \pm 7)$ & $100( \pm 8)$ \\
\hline 1 & $80( \pm 6)$ & $90( \pm 3)$ & $85( \pm 2)$ & $90( \pm 7)$ & $95( \pm 7)$ & $90( \pm 2)$ \\
\hline 2 & $70( \pm 6)$ & $70( \pm 6)$ & $65( \pm 2)$ & $80( \pm 2)$ & $80( \pm 5)$ & $74( \pm 3)$ \\
\hline 4 & $0( \pm 0)$ & $50( \pm 6)$ & $0( \pm 0)$ & $0( \pm 0)$ & $75( \pm 3)$ & $65( \pm 4)$ \\
\hline
\end{tabular}

"Values are the mean of triplicate viable counts. Cells were incubated at $35^{\circ} \mathrm{C}$ at various time intervals on an orbital shaking incubator in RPMI-1640 medium containing various concentrations of alcohols. Viability count was done after $48 \mathrm{~h}$. Values in parenthesis indicate standard deviation. 


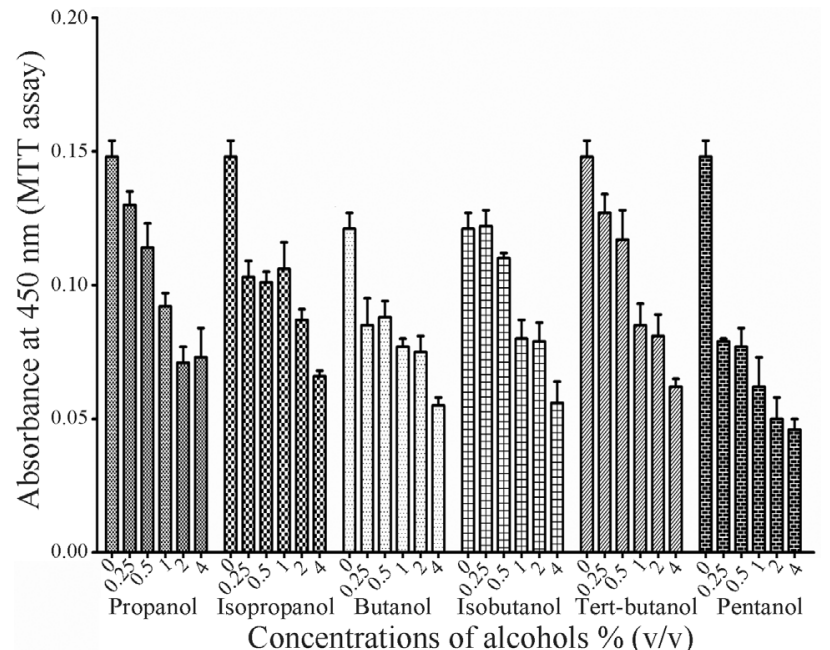

Figure 2 - Effect of various alcohols on biofilm formation by Candida albicans. In vitro biofilm development in the presence of alcohols was quantified by using MTT assay. RPMI-1640 medium containing various concentrations of respective alcohols and one control without alcohol was incubated at $37^{\circ} \mathrm{C}$ for $24 \mathrm{~h}$.
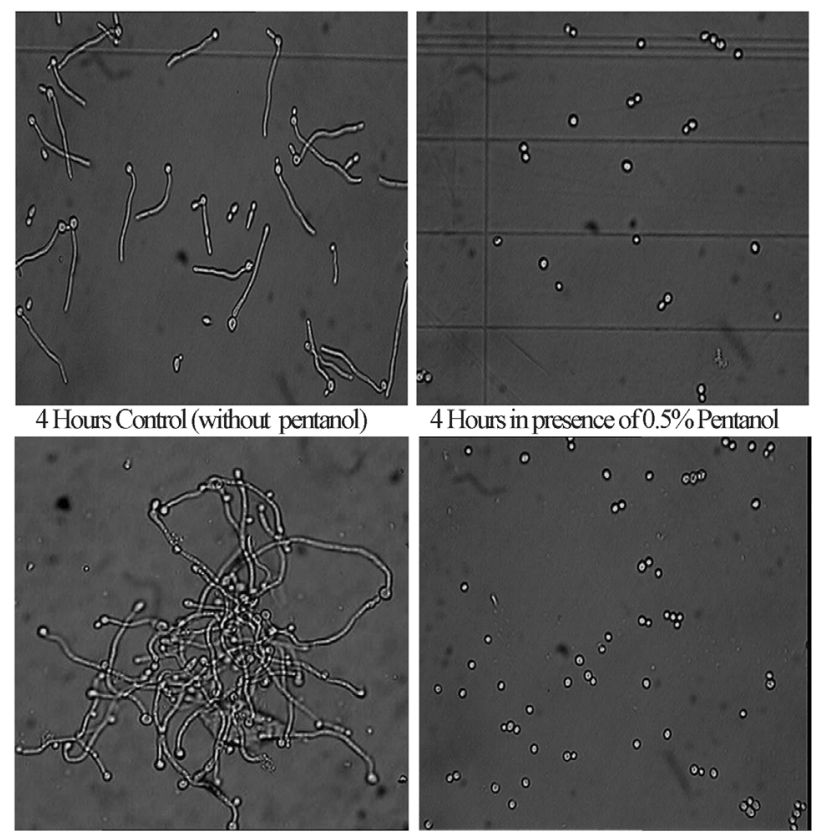

6 Hours Control (without Pentanol)

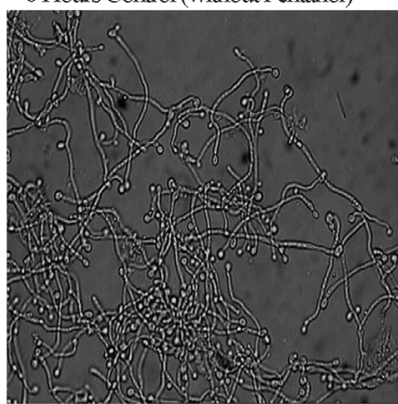

8 Hours Control (without pentanol)
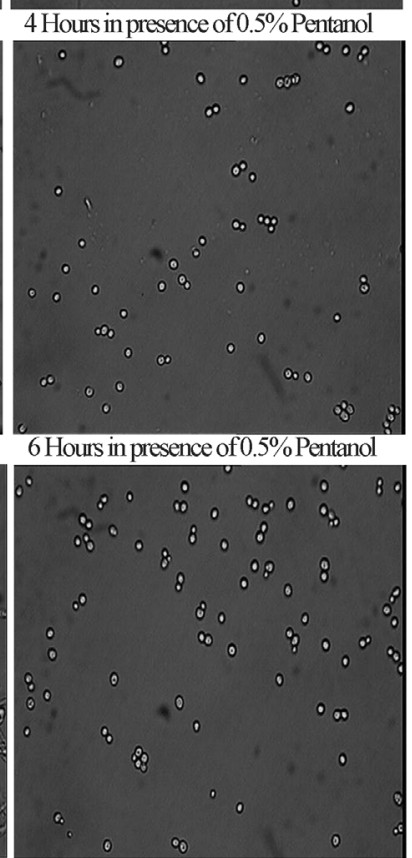

8 Hours in presence of $0.5 \%$ Pentanol

Figure 3 - Morphology of Candida albicans cells in presence of pentanol. Briefly cells were incubated in serum at $37^{\circ} \mathrm{C}$ for 4,6 , and $8 \mathrm{~h}$ respectively and morphology was assessed after incubation and photographs were taken by Labomed imaging device. (Magnification x100).

seated mycosis, and biofilms it may produce considerable amount of alcohols, because of the anaerobic conditions. The quorum sensing molecule, farnesol is undetectable under anaerobic conditions of growth, suggesting signalling roles for other metabolites (Hornby et al., 2011). Alcohols are very common metabolites produced by yeast under anaerobic conditions. Interestingly, the concentrations of al-
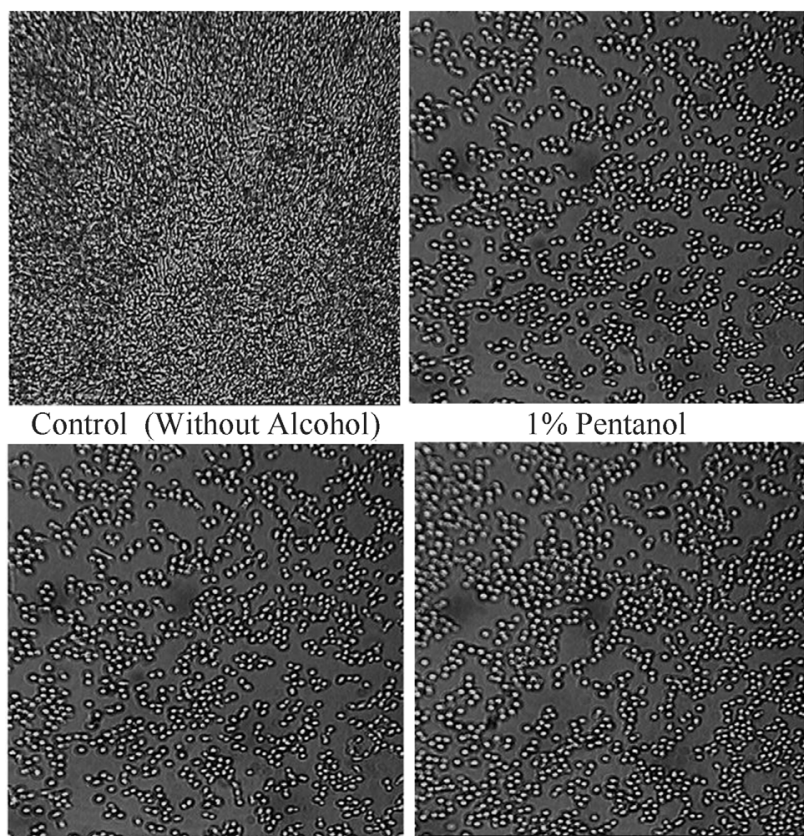

$1 \%$ Pentanol

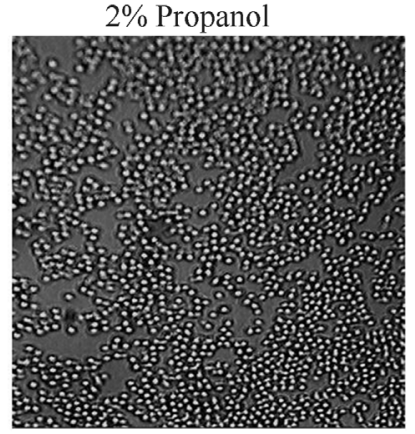

$2 \%$ Butanol

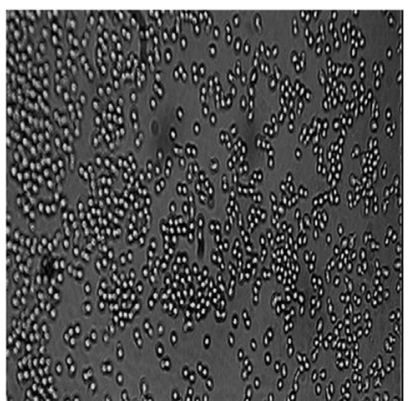

$2 \%$ Tert-butanol

Figure 4 - Candida albicans biofilm development in presence of various alcohols. In vitro biofilms were developed on 96-well polystyrene surfaces in the presence of alcohols and one control without alcohol was incubated at $37^{\circ} \mathrm{C}$ for $24 \mathrm{~h}$. After incubation biofilms were observe microscopically and photographs were taken by Labomed imaging device. (Magnification x200).

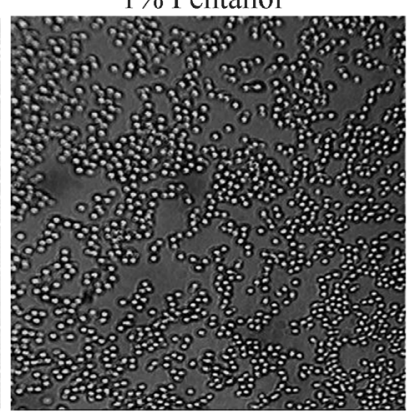

$2 \%$ Isopropanol

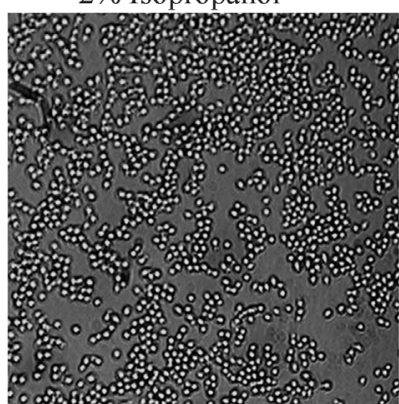

$2 \%$ Isobutanol 
cohols produced under anaerobic conditions are more in comparison to yeast cells produced under aerobic growth (Ghosh et al., 2008). Most of the alcohols tested by us inhibited yeast to hyphal form switching.

We have earlier reported the morphogenetic regulatory properties of ethyl alcohol (Chauhan et al., 2010) and its oxidative product, acetaldehyde in $C$. albicans (Chauhan et al., 2011). In this paper we report for the first time the effect of pentanol, butanol, propanol, isobutanol, isopropanol, and tertiary-butanol on morphogenetic switching, biofilm formation, growth, and viability in $C$. albicans. Among the various alcohols studied, pentanol (amyl alcohol) was found to be very effective. $0.5 \%$ of pentanol completely blocked induced morphogenesis without affecting viability of $C$. albicans. The hyphal inhibitory concentration of pentanol did not alter growth and viability of Candida cells. The same concentration of pentanol showed around $50 \%$ reduction in metabolic activity in biofilm development (Figure 2). While for other alcohols the hyphal inhibitory concentration showed $10-30 \%$ reduction in viability and $35-45 \%$ decreased in metabolic activity was observed in Candida biofilms compared to that of control (Table 2). Based on our results, we have proposed a model for the role of alcohols in C. albicans biofilms (Figure 5).

Deeper regions of mature biofilms may produce anaerobic conditions (Dumitru et al., 2004). Anaerobic conditions also persist in deep seated mycosis. The type of alcohol produced may vary depending on the amino acid availability. For example, availability of leucine, isoleucine, valine, and threonine may favor the production of amyl alcohol, isoamyl alcohol, butanol, and propanol respectively (Dickinson et al., 1998; Lorenz et al., 2000; Hazelwood et al., 2008). The alcohols may exert effects de-

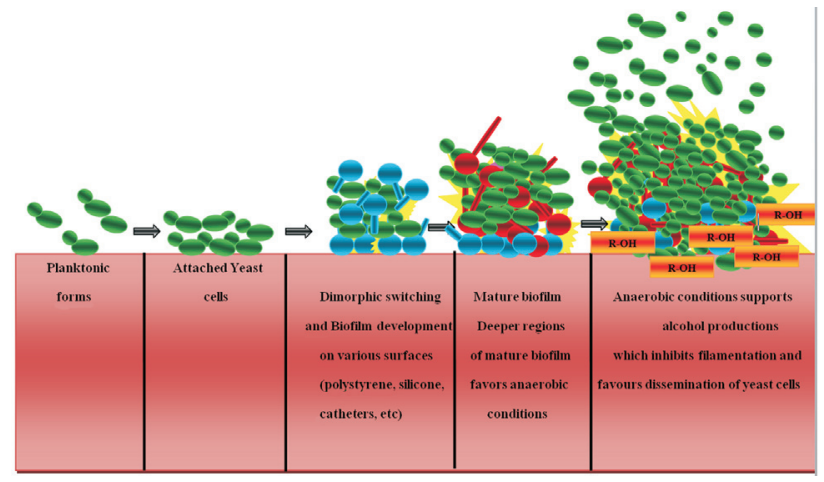

Figure 5 - A model for the role of alcohols favoring disseminative mode of growth in Candida albicans. Planktonic cells get attached to surfaces of polystyrene, catheter, silicone, etc. The adhered cells start to develop biofilms. Biofilm development leads to matured biofilm which include the mixture of yeast cells (green colored), hyphae (red and blue colored) and extracellular polymatrix (yellow colored). Deeper region of matured biofilm favors anaerobic conditions which gives rise to the production of alcohols. These alcohols inhibit filamentation and favors disseminative growth which allows Candida to escape from the alcohol poisoned environment. pending on its concentrations. At lower concentrations like $0.5 \%$ in case of amyl alcohol it may inhibit morphogenetic switching and support yeast phase growth. While at high concentrations it may slow down the growth rate. Most of the alcohols may exert similar effect like ethyl alcohol, amyl alcohol, isoamyl alcohol, etc. Favoring of yeast phase growth may support disseminative mode of growth and also aid to escape from the alcohol poisoned environment. It may also offer ecological advantages for $C$. albicans over other competing organisms which are more susceptible to alcohols than Candida under mixed species of populations.

\section{Acknowledgments}

The authors thank Prof. S. B. Nimse, Honourable Vice Chancellor, SRTM University, Nanded (M.S), India, for encouragement and motivation.

\section{References}

Baillie GS, Douglas LJ (1999) Role of dimorphism in the development of Candida albicans biofilms. J Med Microbiol 48:671-679.

Chauhan NM, Raut JS, Karuppayil SM (2010) A morphogenetic regulatory role for ethyl alcohol in Candida albicans. Mycoses 54:e697-703.

Chauhan NM, Raut JS, Karuppayil SM (2011) Acetaldehyde inhibits the yeast-to-hypha conversion and biofilm formation in Candida albicans. Mycoscience 52:356-360.

Davis-Hanna A, Piispanen AE, Staeva II, Hogan DA (2008) Farnesol and dodecanol effects on the Candida albicans RascAMP signaling pathway, and the regulation of morphogenesis. Mol Biol 67:47-62.

Dickinson JR (2008) Filament formation in Saccharomyces cerevisiae- a Review. Folia Microbiol 53:03-14.

Dickinson JR, Harrison SJ, Hewlins JE (1998) An investigation of the metabolism of valine to isobutyl alcohol in Saccharomyces cerevisiae. The J Biol Chem 273:2575125756.

Dufour N, Rao RP (2011) Secondary metabolites and other small molecules as intercellular pathogenic signals. FEMS Microbiol Letters 314:10-17.

Dumitru R, Hornby JN, Nickerson KW (2004) Defined anaerobic growth medium for studying Candida albicans basic biology and resistance to eight antifungal drugs. Antimicrob Agents Chemother 48:2350-2354.

Fletcher M (1983) Effects of methanol, ethanol, propanol, and butanol on bacterial attachment to surfaces. J Gen Microbiol 129:633-641.

Fridkin SK, Irwin MY (1983) Epidemiology of nosocomial fungal infections. Clin Microbiol Reviews 09:499-511.

Ghosh S, Kebaara BW, Atkin AA, Nickerson KW (2008) Regulation of Aromatic alcohol production in Candida albicans. Appl Environ Microbiol. 74:7211-7218.

Hawser SP, Douglas LJ (1994) Biofilm formation by Candida species on the surface of catheter materials in vitro. Infect Immun 62:915-921.

Hazelwood LA, Daran JM, Maris JA, Pronk JT, Dickinson JR (2008) The Ehrlich pathway for fusel alcohol production: a 
century of research on Saccharomyces cerevisiae. Appl Environ Microbiol 74:2259-2266.

Hornby JB, Jensen EC, Lisec AD, Tasto JJ, Jahnke B, Shoemaker R, Dussualt P, Nickerson KW (2001) Quorum sensing in the dimorphic fungus Candida albicans is mediated by farnesol. Appl Environ Microbiol. 67:2982-2992.

Jean-Marcel S (1998) Candida adherence phenomena, from commensalism to pathogenecity. Int Microbiol 1:117-122.

Lo HJ, Kohler JR, DiDomencia B, Loebenberg D, Fink GR (1997) Nonfilamentous Candida albicans are avirulent. Cell 90:939-949.

Lopez-Ribot JL (2005) Candida albicans Biofilms: More Than Filamentation. Current Biol 15:R453-R455.

Lorenz MC, Cutler NS, Heitman J (2000) Characterization of alcohol-induced filamentous growth in Saccharomyces cerevisiae. Mol Biol Cell 11:183-199.

Martins M, Henriques M, Azeredo J, Rocha SM, Coimbra MA, Oliveira R (2007) Morphogenesis control in Candida albicans and Candida dubliniensis through signaling molecules produced by planktonic and biofilm cells. Eukaryo Cell 6:2429-2436.
Moragues MD, Asturias JA, Sevilla M (1998) Morphogenetic potency of short-chain n-alkanols related to Aureobasidium pullulans. Current Microbiol 16:243-245.

National Committee for Clinical Laboratory Standards. Reference method for broth dilution antifungal susceptibility testing of yeast (1997) Approved standard NCCLS M27-A. National Committee for Clinical Laboratory Standards, Wayne, Pa.

Parahym AMR, Melo LRB, Morais VLL, Neves RP (2009) Candidiasis in pediatric patients with cancer interned in university hospital. Braz J Microbiol 40:321-324.

Saville SP, Lazzell AL, Monteagudo C, Lopez-Ribot JL (2003) Engineered control of cell morphology in vivo reveals distinct roles for yeast and filamentous forms of Candida albicans during infection. Eukary Cell 02:1053-1060.

Virudes A, Peman J, Canton E, Ubeda P, Lopez-Ribot JL, Goberando L (2003) Candidemia at a tertiary care hospital: epidemiology, treatment, clinical outcome, and risk factors for death. Eur J Clin Microbiol Infect Dis 01:767-774.

Yang Y (2003) Virulence factors of Candida species. J Microbiol Immunol Infect 36:223-228.

All the content of the journal, except where otherwise noted, is licensed under a Creative Commons License CC BY-NC. 